\title{
Silent Acute Coronary Syndrome in a Mild Carbon Monoxide Poisoning
}

\author{
Mustafa Mahmood Eid \\ Emergency Department, Al Ain Hospital, United Arab Emirates
}

\section{ABSTRACT}

Introduction: Carbon monoxide ( $\mathrm{CO}$ ) poisoning continues to be challenging in diagnosis and management. The complications related to CO poisoning are variable and unpredictable. Case presentation: We present the case of a 73-year-old woman who was involved in an indoor fire. Although she was asymptomatic and had a low CO level, she developed an acute coronary syndrome. The condition of the patient recovered remarkably after treatment with oxygen supplementation and anti-ischemic drugs. Conclusion: This case indicates that an acute coronary syndrome could represent a complication of CO poisoning even in asymptomatic patients with low levels of $\mathrm{COHb}$.

Keywords: carbon monoxide poisoning, acute coronary syndrome, myocardium, inhalation, wounds and injuries

\section{ARTICLE HISTORY}

Received: March 22, 2020

Accepted: May 13, 2020

\section{CORRESPONDENCE}

Mustafa Mahmood Eid

Shakboot Bin Sultan Street

Al Ain / U.A.E.

P.O. Box 1006

United Arab Emirates

Tel: +971505848181

E-mail: dr.mustafa191982@gmail.com

\section{INTRODUCTION}

In patients with carbon monoxide ( $\mathrm{CO}$ ) poisoning, the history of the present illness is tremendously variable and inconsistent. ${ }^{1}$ Symptoms are often vague and may appear as emphasized constitutional complaints. ${ }^{2}$ The neurologic consequences of $\mathrm{CO}$ poisoning have been well described. However, the cardiovascular consequences of CO poisoning are limited to isolated case reports. Besides, previous studies have focused on the cardiac dysfunction related to CO poisoning and indicated that myocardial injury is not uncommon in moderate-to-severe $\mathrm{CO}$ poisoning, while no studies documented, so far, the effect of mild CO poisoning. ${ }^{3,4}$

The present manuscript aims to present a rare case of asymptomatic acute coronary syndrome (ACS) occurring after mild carbon monoxide poisoning. We present the case of a patient with $\mathrm{CO}$ poisoning who was asymptomatic and only had external signs of smoke inhalation, and in whom an ACS was subsequently diagnosed, most prob- ably caused by development of demand ischemia, coronary spasm, or direct CO toxic effect. ${ }^{3}$

\section{CASE PRESENTATION}

A 73-year-old woman, with hypertension and hyperthyroidism, came to the emergency department after a fire started in her house due to an air conditioning problem. The patient was brought by an ambulance within 30 minutes of the incident with her clothes, face, and whole body blackish in color. She did not present dyspnea or any respiratory distress.

On examination, the patient was hemodynamically stable. Her nose and mouth contained smoke particles. There was no hoarseness, stridor, or choking. Her chest was clear with no extra heart sounds.

Therefore, the patient was cleaned from all the smoke and dust. In addition, an arterial blood gas (ABG) test on room air was executed, as well as other blood tests. Also, an ECG (Figure 1) and chest X-ray (Figure 2) were per- 


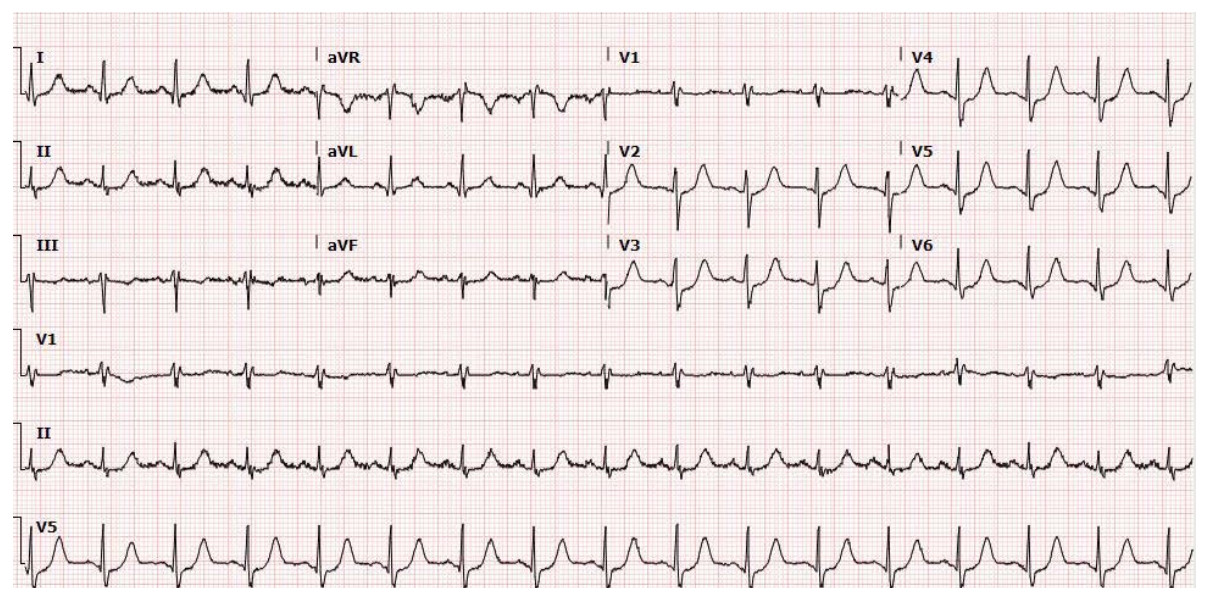

FIGURE 1. Twelve-lead ECG tracing performed in the Emergency Department

formed, and the patient was started on oxygen. Her ECG revealed sinus rhythm with a left anterior fascicular block (LAFB) and a right bundle branch block (RBBB), while the chest $\mathrm{X}$-ray showed bilateral hilar congestion and accentuated vascular markings.

Consequently, the patient felt better and asked to go home. Her ABG showed a COHb of $5.9 \mathrm{mmol} / \mathrm{L}$ (normal 0.0-3.0 mmol/L) and lactate of $2.8 \mathrm{mmol} / \mathrm{L}$ (normal 0.5$1.8 \mathrm{mmol} / \mathrm{L}$ ). Her blood tests (Table 1) were within normal limits apart from troponin-T, which was $0.061 \mathrm{\mu g} / \mathrm{L}$ (nor$\mathrm{mal}<0.015 \mu \mathrm{g} / \mathrm{L}$ ).

Thus, the medical team was consulted to evaluate the patient. They assumed that a cardiac issue is unlikely as the patient was symptoms free, had no complaints, and her $\mathrm{COHb}$ level was low. Nevertheless, since the patient had ECG changes without any previous ECG available for

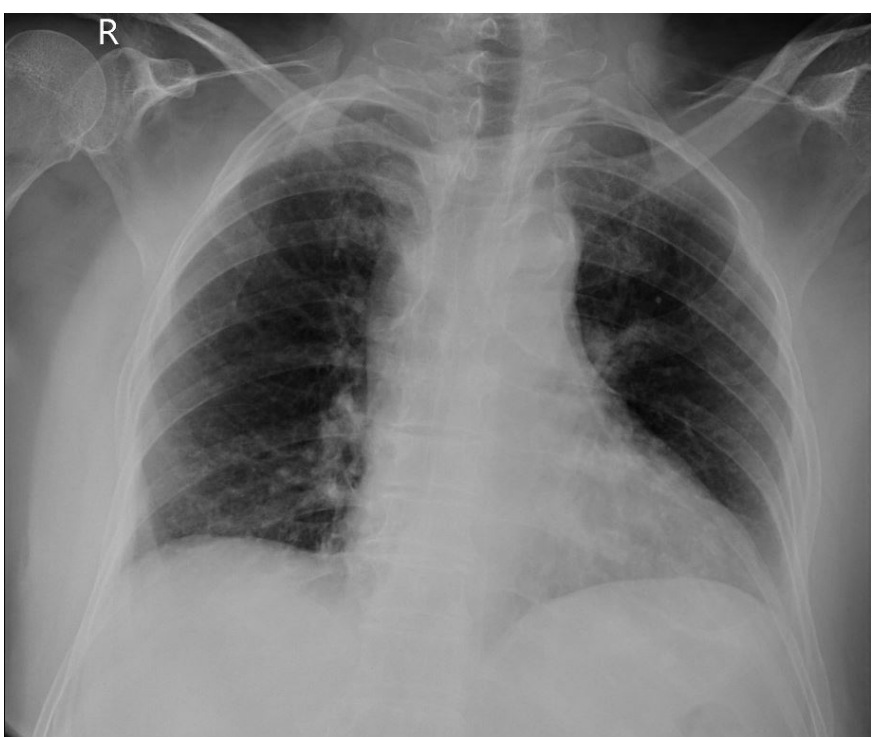

FIGURE 2. Antero-posterior chest $X$-ray imaging of the patient comparison, the troponin determination was repeated after 3 hours and was found to be elevated up to $0.35 \mu \mathrm{g} / \mathrm{L}$, even though the patient was comfortable and wishing to leave against medical advice. As a result, the patient was admitted to the coronary care unit, where oxygen was supplied and a full ACS protocol was initiated. After 3 days, the patient was discharged home. Her troponin level came down and her echocardiography confirmed mild concentric left ventricular hypertrophy. No coronary angiography was conducted for the patient as per the cardiologist's opinion.

The patient consented to the publication of her data.

\section{DISCUSSIONS}

CO intoxication is a paramount, potentially lethal, often overlooked, and often scantily treated medical emergency. A study conducted on this topic revealed that about $30 \%$ of CO poisonings were inadequately assessed at first contact with the health system. ${ }^{5}$ Furthermore, patients suffering from $\mathrm{CO}$ poisoning show a range of clinical symptoms that vary between some dizziness and a headache, and the extreme of a coma and possibly death. ${ }^{6}$

In mild and moderate $\mathrm{CO}$ intoxication, symptoms like headache, vomiting, vertigo, confusion, chest pain, or shortness of breath can appear, while in severe cases, myocardial injury, seizure, and coma can occur. ${ }^{7}$

In our case, although the patient was considered to have a mild intoxication with no symptoms, she was found to have an ACS. ACSs are rare presentations in acute carbon monoxide poisoning. Therefore, the American College of Physicians recommends performing an electrocardiogram and evaluation of cardiac biomarker levels in patients with moderate to severe CO poisoning. ${ }^{8}$

$\mathrm{CO}$ toxicity compromises and damages the process of 
TABLE 1. Blood tests result of the patient indicating elevated troponin levels

\begin{tabular}{lcc}
\hline Blood tests & Results & Normal range \\
\hline WBC & $10.2 \times 10^{9} / \mathrm{L}$ & $4-11 \times 10^{9} / \mathrm{L}$ \\
Hemoglobin & $15.5 \mathrm{~g} / \mathrm{dL}$ & $11.5-16.5 \mathrm{~g} / \mathrm{dL}$ \\
Platelets & $230 \times 10^{9} / \mathrm{L}$ & $140-440 \times 10^{9} / \mathrm{L}$ \\
C-reactive protein (CRP) & $2 \mathrm{mg} / \mathrm{L}$ & $0-5 \mathrm{mg} / \mathrm{L}$ \\
Troponin-T & $0.061 \mu \mathrm{g} / \mathrm{L}$ & $<0.015 \mu \mathrm{g} / \mathrm{L}$ \\
pH & 7.41 & $7.35-7.45$ \\
Cyanide level & $0.05 \mu \mathrm{gg} / \mathrm{mL}$ & $<0.2 \mu \mathrm{g} / \mathrm{mL}$ \\
Urea and electrolytes & Within normal limits & \\
\hline
\end{tabular}

delivering and utilizing oxygen in the cells. Furthermore, various and numerous areas in the body are affected by CO; however, the most impacted organs are those that require the greatest oxygen levels (like the heart and brain). In addition, because $\mathrm{CO}$ has a higher affinity to cardiac myoglobin than hemoglobin, it binds more strongly to it, which results in hypotension and myocardial depression. These further aggravate hypoxia in the tissues. Moreover, inflammatory or immunological damage due to direct changes at a cellular level can be provoked by CO. There are many different mechanisms for this such as binding to intracellular proteins, nitric oxide generation, or mitochondrial oxidative stress. ${ }^{9}$

Patients with CO poisoning commonly suffer myocardial injuries that are acute in nature and are usually linked with an escalation in mortality rates in the long term. A retrospective study of 230 patients with moderate or severe CO poisoning referred to a specialized center found evidence of myocardial ischemia (characteristic electrocardiographic changes or elevated serum cardiac biomarkers) in one-third of the cases. ${ }^{10}$

The same cohort of patients underwent re-examination and long-term follow up (the median number of years was 7.6), and it was found that those who had endured an acute myocardial injury had a $24 \%$ death rate. Furthermore, it was observed that the mortality rates of the patients who had myocardial injuries were double to the mortality of those patients with CO poisoning who did not show any signs of injury. Additionally, it is approximated that the mortality of CO poisoned patients who present associated myocardial injury is three times higher than the mortality of a similar group of patients not exposed to CO. ${ }^{11}$

\section{CONCLUSION}

This unusual and rare case underlines the need to conduct cardiac enzymes tests and ECG, in addition to other necessary cardiovascular examinations, in patients suffering from acute and severe CO poisoning. Moreover, a physician should always bear in mind that $\mathrm{CO}$ poisoning can happen to any burn patient even if he/she is asymptomatic; therefore, the physician must look for possible complications related to $\mathrm{CO}$ poisoning, including cardiac complications. The case illustrated represents a very rare, but possibly life-threatening condition, occurring in a patient suffering a CO intoxication.

\section{CONFLICT OF INTEREST}

Nothing to declare.

\section{REFERENCES}

1. Harper A, Croft-Baker J. Carbon monoxide poisoning: undetected by both patients and their doctors. Age Ageing. 2004;33:105-109. doi: 10.1093/ageing/afho38.

2. Tomaszewski C. Carbon monoxide poisoning. Early awareness and intervention can save lives. Postgrad Med. 1999;105:3950. doi: 10.3810/pgm.1999.01.496.

3. Lee FY, Chen WK, Lin CL, et al. Carbon monoxide poisoning and subsequent cardiovascular disease risk: a nationwide population-based cohort study. Medicine (Baltimore). 2015;94:e624. doi: 10.1097/MD.0000000000000624.

4. Satran D, Henry CR, Adkinson C, et al. Cardiovascular Manifestations of Moderate to Severe Carbon Monoxide Poisoning. J Am Coll Cardiol. 2005;45:1513-1516. doi: 10.1016/j. jacc.2005.01.044.

5. Barrett L, Danel V, Faure J. Carbon monoxide poisoning, a diagnosis frequently overlooked. J Clin Toxicol. 1985;23:309313. doi: 10.3109/15563658508990638.

6. Rose JJ, Wang $\mathrm{L}, \mathrm{Xu} \mathrm{Q}$ et al. Carbon Monoxide Poisoning: Pathogenesis, Management, and Future Directions of Therapy. Am J Respir Crit Care Med. 2017;195:596-606. doi: 10.1164/ rccm.201606-1275CI.

7. Kao LW, Nañagas KA. Carbon monoxide poisoning. Emerg Med Clin N Am. 2004;22:985-1018. doi:10.1016/j.emc.2004.05.003.

8. Wolf SJ, Maloney GE, Shih RD, et al. Clinical Policy: Critical Issues in the Evaluation and Management of Adult Patients Presenting to the Emergency Department with Acute Carbon Monoxide Poisoning. Ann Emerg Med. 2017; 69:98-107.e6. doi: 10.1016/j.annemergmed.2016.11.003.

9. Hampson NB, Piantadosi CA, Thom SR, et al. Practice recommendations in the diagnosis, management, and prevention of carbon monoxide poisoning. Am J Respir Crit Care Med. 2012;186:1095-1101. doi: 10.1164/rccm.2012071284 CI.

10. Satran D, Henry CR, Adkinson C, et al. Cardiovascular manifestations of moderate to severe carbon monoxide poisoning. J Am Coll Cardiol. 2005;45:1513-1516. doi: 10.1016/j. jacc.2005.01.044.

11. Henry CR, Satran D, Lindgren B, et al. Myocardial injury and long-term mortality following moderate to severe carbon monoxide poisoning. JAMA. 2006;295:398-402. doi: 10.1001/ jama.295.4.398. 